\title{
Mitigation of the ripple effect in supply chains: Balancing the aspects of robustness, complexity and efficiency
}

\author{
Judit Monostori ${ }^{\mathrm{a}, \mathrm{b}, *}$ \\ ${ }^{a}$ Centre of Excellence in Production Informatics and Control, Institute for Computer Science and Control, Eötvös Loránd Research Network, Budapest, Hungary \\ ${ }^{\mathrm{b}}$ Department of Material Handling and Logistics Systems, Budapest University of Technology and Economics, Budapest, Hungary
}

A R T I C L E I N F O

\section{Article history:}

Available online 15 February 2021

\section{Keywords:}

Supply chains

Distribution networks

Robustness

Complexity

Efficiency

Ripple effect

\begin{abstract}
A B S T R A C T
The robustness of supply chains, i.e. their ability to cope with external and internal disruptions and disturbances, is of vital importance. The complicated interrelationships of supply chains' robustness, complexity and efficiency are outlined and analyzed in the paper. Aiming to balance these aspects and to compare different supply chain settings in a reliable way, measures are provided for characterizing both structural and operational robustness / complexity of supply chains. A methodology and a framework for the holistic evaluation of supply chains' robustness, complexity and efficiency are also highlighted. Their applicability is illustrated by the results of a case study on distribution networks, indicating that the three aspects can be balanced while mitigating the ripple effect.
\end{abstract}

(C) 2022 The Author. This is an open access article under the CC BY-NC-ND license (http:// creativecommons.org/licenses/by-nc-nd/4.0/).

\section{Introduction}

Until recently the efficiency aspects of production were put into the foreground, as to be considered and strived for, sometimes even exclusively. The vulnerability of production structures received much less attention, and consequently, by now, it is usually beyond its acceptable degree. The frequently changing and uncertain environment which manufacturing companies are facing nowadays, requires robustness at every level of the production hierarchy, including the level of supply chains and networks. The COVID-19 pandemic gave fresh momentum to the research activities related to supply chains' robustness [1-4]. In the cyber-physical era [5], the complexity of supply chains may increase in parallel with the opportunity to realize more robust systems [6]. However, the question arises, what level of complexity is required to achieve a certain degree of robustness while, naturally, keeping the efficiency aspects in mind as well [7]. In other words, how to balance the aspects of robustness, complexity and efficiency.

These questions represent scientific challenges and their answering may be of significant interest to the production industry. Beyond the importance of the topic, the result of an extensive literature survey gave additional motivations for the research:

\footnotetext{
* Correspondence to: Institute for Computer Science and Control, Kende u. 13-17, Budapest 1111, Hungary.

E-mail address: mesterne.monostori.judit@sztaki.hu (J. Monostori).
}

- Most of the related papers deal either with the robustness or the complexity of supply chains, and only relatively few publications can be found which jointly assess them. In this regard, the intentions were to make a contribution to this line of research addressing a more comprehensive treatment of supply chains, moreover, to consider the aspects of robustness, complexity and efficiency together.

- The investigations rarely focus on the structural and the operational robustness and/or complexity at the same time. In this respect, the plans were to make a clear distinction between the two kinds and to exploit their complementary natures in improving the performance of supply chains.

The main aims of the paper are to explore the complicated interrelationships of supply chains' robustness, complexity and efficiency, to underline the importance of striving for an appropriate balance between them, and to show that the search for balanced solutions is not a hopeless undertaking.

All the above are reflected in the structure of the paper.

Section "Key concepts and challenges" outlines some key concepts and challenges based on a comprehensive literature analysis of previous works, such as risk categories, bullwhip and ripple effects, robustness and connected terms, the conflicts between efficiency and robustness, and the relation of complexity and robustness. The timeliness of the research, i.e. handling supply chains' robustness and complexity together, considering both structural and operational points of view, not forgetting efficiency either, is justified by identifying the research gaps in this field. 
In Section "Quantitative measures of supply chains' robustness and complexity", graph theoretical measures are given for characterizing the structural robustness and complexity of supply chains and networks. Measures for assessing their operational robustness and complexity that can be determined by statistical methods and/or by simulations are also highlighted.

Section "Methodology and framework for the evaluation of supply chains' robustness, complexity and efficiency" introduces a methodology and a framework for the quantitative evaluation of supply chains' robustness, complexity and efficiency.

A case study on distribution networks is described in Section "A case study on distribution networks", indicating that the envisioned balance between the three aspects can be achieved while mitigating the ripple effect.

Finally, Section "Conclusions" outlines the main messages of the paper, summarizes the results, underlines their scientific and practical relevancies, and indicates the planned further investigations.

\section{Key concepts and challenges}

A vast number of publications dealing with different aspects of the design and management of supply chains and networks can be found in the literature. The main goals of the section are to introduce the most important concepts related to the topic of the paper, and to clarify in what sense they will be used hereinafter, and, furthermore, to point out the main challenges to be tackled and to identify the research gaps addressed in the work to be reported on.

\section{Main risks the supply chains face}

Supply chains are exposed to risks of various types. Demandside, supply-side and catastrophic risks are differentiated in [8].

Demand-side risks include deviations of the actual demands from the forecasted ones, inadequate supply chain coordination, and problems in the products' physical distribution. Possible negative effects of demand-side risks are inefficient capacity utilization, costly shortages or surpluses.

Supply-side risks involve suppliers' unreliability, capacity constraints, changes in the product design or in the technology, quality problems of the supply, and weak logistics performance. Typical negative consequences of supply-side risks are backlogs, late deliveries, and inappropriate functioning of some elements / parts of the supply chains concerned, resulting, many times, in lower level of overall performance.

Catastrophic risks include natural hazards (e.g. volcanic eruptions, hurricanes, earthquakes, tsunamis, floods and droughts), economic crises, social-political instabilities, civil unrests, and even acts of terrorism. Because of the often geographically dispersed nature of supply chains, local problems can significantly affect even remote parts of them, negatively influencing the performance of entire supply chains.

Obviously, the occurrence probability and the potential impact of the given risk are important characteristics. Their product is a widely used measure for risks' ranking [9]. Nowadays, two distinct risk categories represent special challenges for the researchers: the recurrent (sometimes called operational) risks and the disruptive ones, i.e. frequent events with low impact and rare events with high impact, respectively $[10,11]$.

\section{Bullwhip effect, ripple effect}

The bullwhip effect $[12,11]$, i.e. the amplification of the demand volatility in the upstream direction of the supply chain, is well known for researchers and practitioners of the field.
In contrast to the bullwhip effect, the ripple effect [13,14], which arises from disruptions at the supply chain elements, generates relatively novel challenges for supply chain managers. Disruptions' negative effects may ripple through the supply chain mainly in the downstream direction, moreover, they can spread to other supply chains as well.

The bullwhip and ripple effects are related to the recurrent and disruptive risk categories (see Subsection "Main risks the supply chains face"), respectively. As typical risks, e.g. demand fluctuation in the former, and plant unavailability or severe strikes in the latter one can be mentioned. Both effects can influence critical parameters of the supply chains, e.g. inventory shortages, increased lead times and lost sales may occur. In order to recover from the situation, usually short-term coordination actions for balancing demand and supply are initiated as reaction to the bullwhip event. Fighting against the ripple effect mostly requires middle- and long-term coordination actions and investments.

When dealing with ripple effects, which belong to the disruptive risk category, a particular difficulty arises, i.e. their occurrence probabilities and the magnitudes of their potential consequences are hard to estimate, because little or no empirical knowledge is available about them in a given supply chain.

\section{Robustness, resilience and vulnerability of supply chains}

The concept of robustness - the word comes from the Latin robustus, meaning strong - appears in different disciplines [6], e.g. in architecture, economics, biology, computer science, systems and control science, and - naturally - in mathematics (e.g. robust optimization).

As to the robustness of supply chains, various, partly overlapping, partly even contradictory definitions are given in the literature. Some examples are:

- "The ability of a network to cope with changes in the competitive environment without resorting to changes in the network structure" [15].

- "The system's ability to resist an accidental event and return to do its intended mission and retain the same stable situation as it had before the accidental event" [16].

- "The ability of a supply chain to maintain a given level of output after a failure" [17].

- "The ability of a supply chain network to carry out its functions despite some damage done to it, such as the removal of some of the nodes and/or links in the network" [18,19].

A more comprehensive enumeration and comparison of definitions of supply chains' robustness can be found, e.g. in [20].

Another important concept related to the previous one is the resilience of supply chains. The word comes from the Latin resilio, meaning to rebound. The concept is adopted from the material sciences, where it characterizes materials' ability to recover their original shapes following a deformation. In case of supply chains, it represents their ability to, and the speed at which they can, return to their normal performance levels following a disruption [21].

In the supply chain literature, similarly to the robustness, a variety of resilience definitions are given, emphasizing different aspects, as one can see from the following examples:

- "The ability of a system to return to its original state or move to a new, more desirable state after being disturbed" [22].

- "The adaptive capability of the supply chain to prepare for unexpected events, respond to disruptions, and recover from them by maintaining continuity of operations at the desired level of connectedness and control over structure and function" [23]. 
- “The supply chain's ability to react to the negative effects caused by disturbances that occur at a given moment in order to maintain the supply chain's objectives" [24].

Though, when comparing robustness and resilience, numerous authors underline the adaptation ability of resilient systems as a distinguishing feature, the real situation is not so clear-cut (see a multitude of resilience definitions in review papers, e.g. $[25,26]$ ).

Additionally, other similar concepts, e.g. agility, responsiveness, flexibility and changeability, to mention only some of them, are also in use [27-30]. All, in some respects, relate to the ability of a system to accommodate perturbations without losing functionality.

Without examining in detail the differences between the formulations given even for the same concept in the literature, and the overlaps between the various ones, in the paper the term robustness will be mostly used, with the following comprehensive formulation: "In the general sense, a supply chain is robust if it is able to comply with the most important key performance indicators (KPIs) set towards it, at an acceptable level (i.e. remaining in a predefined robustness zone) during and after unexpected event(s)/ disruption(s) which caused disturbances in one or more production or logistics processes" [7].

Fig. 1 (a further developed version of the figure in [31]) illustrates this concept, also indicating the possible outcome when the new stable state resumes with an even higher KPI.

Naturally, not only one KPI can be influenced by a given disruption, moreover, the time that is required to reach an acceptable new stable state (disruption or recovery time) can depend on which KPI is taken into account.

The vulnerability [31] is considered in the paper as a concept closely but inversely related to robustness, i.e. the more vulnerable a supply chain, the less robust it is. In this respect, the main drivers which act against the robustness of supply chains are as follows [8]:

- Customer dependence: Dependence on a dominant customer with a significant proportion of the sales volume. In the presence of a disruption at the dominant customer, the seller firm may be seriously impacted.

- Supplier dependence: Dependence on a dominant supplier to which there are only few alternatives. The severity of a disruption at the dominant supplier for the buyer firm is fundamentally determined by the criticality of the item(s) to be purchased.

- Supplier concentration and single sourcing: Concentrating the sourcing on a relatively small number of suppliers or even on a single one, the company weakens its ability to involve alternative suppliers in critical situations.

- Global sourcing: Globe-spanning supply chains may be faced with increased uncertainty and poorer transparency.

\section{Efficiency versus robustness of supply chains}

For most of the companies efficiency is the ultimate goal. As an obvious consequence, their operations are streamlined by applying management concepts such as outsourcing, lean, just-in-time and just-in-sequence. Low level safety stocks are usually aimed at, and this way, supply chains become vulnerable to different turbulences [7].

Efficiency, on the one hand, and robustness, on the other, drive supply chain managers in mostly opposite directions (Table 1 ).

From Table 1 the conclusion can be drawn that efficiency and robustness pose contradictory requirements towards supply chain design and management. However, the real challenge is to balance

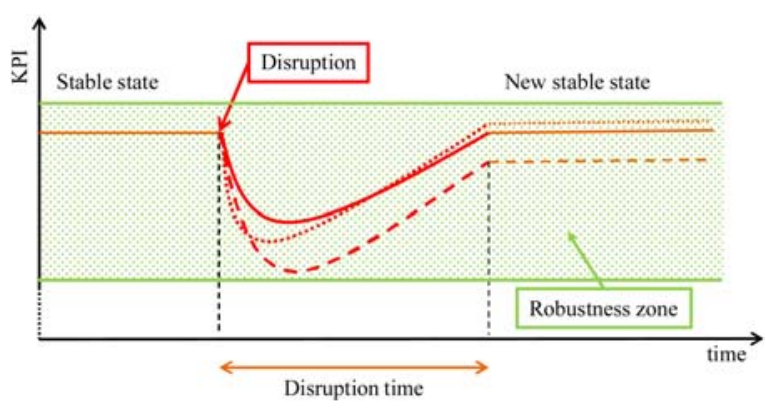

Fig. 1. Delineation of supply chains' robustness used in the paper [7].

these opposing characteristics with the aim of a kind of reconciliation. The feature which incorporates both resilience / robustness and efficiency aspects in supply chain management is denoted as resileanness in [32]. According to this vision, digital technologies and smart operations can contribute to the integration of resilience / robustness and lean thinking.

The increase of supply chains' resilience / robustness usually implies some additional costs. As it is underlined in [33], unnecessary overinvestment in corresponding capabilities can erode profits, therefore, a balanced resilience is to be strived for in supply chains to match their portfolio of capabilities to their pattern of vulnerabilities.

\section{Complexity issues in supply chains}

The complexity and the question, how to handle it came into the foreground at every level of the production hierarchy, thus also at the level of supply chains and networks [34,35].

Three dimensions of the supply base complexity of a focal company are outlined in [36]: the number of suppliers, the degree of differentiation among these suppliers (concerning operational practices, cross-border barriers, technical capabilities), and the level of interrelationships between them. The main statement of the referred paper is that although the reduction of the supply base complexity may be cost-efficient, its incautious implementation can negatively impact the focal company's overall competitiveness.

One possible method to cluster supply chains' complexity is to distinguish between necessary and unnecessary complexities, on the one hand, and between current and potential complexities, on the other [37]. The complexity that provides a distinct competitive advantage and that the market / customer is willing to pay for is considered as necessary complexity, while unnecessary complexity - though usually involves additional costs - cannot offer such benefits. Potential complexity, in contrast to the current one, does not exist at the present time, but may occur in the future. To the different clusters various approaches can be ordered, such as to manage, reduce / eliminate, or to prevent. Necessary complexity needs to be managed, independently of its relation to time (current or potential). Unnecessary, current complexity requires intervention to reduce or even eliminate it as soon as possible. In case of unnecessary, potential complexity, preventive actions may be taken.

Another, highly important categorization is to be mentioned that is applicable in respect of both complexity and robustness of supply chains, namely the structural (static) and the operational (dynamic) complexity / robustness. When investigating a supply chain from structural point of view, the focus is on its elements and the connections between them. While analyzing operational aspects, the dynamic processes occurring in the supply chain are dealt with, considering unchanged structure [38-40]. The two kinds of complexity and robustness, i.e. the structural and the 
Table 1

Characteristics of efficient and robust supply chains (based on [32]).

\begin{tabular}{|c|c|c|}
\hline Criteria & Efficient supply chains & Robust supply chains \\
\hline Primary goal & Supply demand with maximum profit / at minimum cost & Ensure demand fulfillment also in case of disruptions \\
\hline Network organization & Centralized, global & Decentralized, local, diversified, segmented \\
\hline Product design strategy & $\begin{array}{l}\text { Standardization, performance maximization at minimum } \\
\text { product cost }\end{array}$ & $\begin{array}{l}\text { Postponement to ensure product flexibility, product } \\
\text { substitution, capacity pooling }\end{array}$ \\
\hline Pricing strategy & Lower margins because price is a prime customer driver & Potentially higher prices caused by the cost of robustness \\
\hline Manufacturing strategy & Cost reduction through high utilization & Capacity reserves for unforeseen events / disruptions \\
\hline Inventory strategy & Cost reduction through inventory minimization & $\begin{array}{l}\text { Inventory reserves for mitigating risks' potential } \\
\text { consequences }\end{array}$ \\
\hline Lead time strategy & Lead time reduction, but not at the expense of cost increase & Lead time reserves for handling uncertainties \\
\hline Sourcing strategy & Supplier selection based on cost and quality, single sourcing & $\begin{array}{l}\text { Supplier risk exposure analysis, backup suppliers and } \\
\text { multiple sourcing }\end{array}$ \\
\hline
\end{tabular}

operational ones, are strongly interrelated. The behavior of the whole system originates from both.

Naturally, by changing structural and/or operational properties of supply chains, their performance - so also their robustness - can be influenced. Generally, it may be expected that - in tendency - a well-aimed increase of the complexity should initiate similar changes in the robustness. However, unnecessary complexity is to be avoided. In this respect, the challenge - sometimes even the art - is to secure the required level of robustness with the lowest possible level of complexity [7].

Some previous attempts to consider supply chains' robustness and/or complexity

In the literature, especially in the past ten years or so, growing number of papers have been published dealing with robustness and/or complexity of supply chains.

The robustness of supply chains is assessed and the supply chain vulnerability index (SCVI) based on graph theoretical considerations is introduced in [41]. The randomized local rewiring (RLR) approach is presented in [42] for robustness evaluation of original and modified (rewired) distribution networks. It is pointed out that the robustness of the investigated distribution networks can be significantly affected by appropriate changes in their topologies. The graph theoretical approaches are characteristic in numerous publications as well, e.g. in [43-46].

A significant portion of the papers focusing on complexity of supply chains propose information theoretical considerations, i.e. to associate supply chains' complexity with the expected amount of information needed to describe their states. Entropy-related assessment of complexity is the frequent method in this line, see e.g. [47-49,35,39].

A promising approach is to consider supply chains as complex adaptive systems (CASs). The underlying assumption of CASs, a paradigm for analyzing the structure and dynamics of large systems, is that the adaptability of systems creates, but at the same time, also resolves complexity. A CAS is, in fact, a multi-agent system in which "a major part of the environment of any given adaptive agent consists of other adaptive agents, so that a portion of any agent's efforts at adaptation is spent adapting to other adaptive agents" [50]. Supply networks are recognized as CASs, because they are emerging, dynamic, self-organizing and evolving [51-53]. For managing systems of this type, appropriate balances between control and emergence [51], on the one hand, and between simulation and theory [52], on the other, are to be strived for.
Network science is also of high relevance when addressing complexity of supply chains and networks [54,55]. Topological classes of assembly supply chains are introduced in [56].

Relatively few papers can be found which jointly assess supply chains' robustness and complexity, as it is indicated in [57].

Three supply chain design characteristics, namely density, complexity and node criticality, are identified in [58]. Density relates to the geographical positioning of nodes within the supply chain, which can be measured, e.g. by the average distance between them. Complexity is considered as the sum of the number of nodes and the number of connections in the supply chain. Node criticality is the importance of a node, which is context-specific and relative to the importance of other ones within the supply chain. In the referred paper, qualitative propositions are formulated, concerning the influence of the above design characteristics on the severity of supply chain disruptions.

In [59] the examination of complexity in light of robustness, adaptability (flexibility) and economic performance is identified as an important future direction. In [60] a multi-product, multiperiod mixed integer linear programming (MILP) model is used for analyzing the effects of various disruptions on eleven indicators in five supply chains with different complexities. In an empirical study [61], relationship between the supply chains' structural complexity and the frequency of supply-side disruptions is found.

A complex network approach is introduced in [7] for the structural characterization of supply chains and networks from both robustness and complexity points of view. Its feasibility is demonstrated on three types of structures, i.e. on real (industrial) and artificially generated ones, and on structures taken from the literature. Measures for operational robustness are also described and the concept of a framework for evaluating supply chains' robustness, complexity and efficiency is outlined in [6].

An approach to achieve trade-offs between the economic (e.g. profit) and the environmental (e.g. $\mathrm{CO}_{2}$ emission) aspects of supply chains' sustainability is introduced in [62]. It is shown how relatively minor relaxations of the expected profit can lead to supply chain structures not only with reduced transportationrelated $\mathrm{CO}_{2}$ emission, but also with increased structural robustness and complexity.

The present paper can be considered as a next step in the process of handling robustness and complexity issues in supply chains jointly. The developed methodology and framework are not restricted to structural aspects only, but can deal also with operational ones, naturally not neglecting efficiency either. Their applicability is illustrated by the results of a case study focusing on the mitigation of the ripple effect in distribution networks. 


\section{Quantitative measures of supply chains' robustness and complexity}

In order to compare different supply chain settings from robustness, complexity and efficiency points of view, the use of objective measures - if possible, quantitative ones - is of fundamental importance. For efficiency there are some generally accepted measures in use, e.g. the profit or the total cost, however this is not the case for robustness and complexity. In this section, such measures are defined partly based on earlier papers of the author [7,6]. The methodology, the related framework and the investigations described in the subsequent parts rely on these measures. Their enumeration and exact formulations may significantly enhance the readability of the paper.

The measures are introduced in the following order:

- Structural measures of supply chains, in respect of both complexity and robustness.

- Operational measures of supply chains, also from complexity and robustness points of view.

\section{Structural measures of supply chains}

The application of graph theoretical concepts is reasonable to characterize the structural properties of supply chains and networks. The elements (e.g. customers, distribution centers, factories, suppliers) of a chain / network can be modeled by the vertices (nodes) of a graph, while the connections between the elements (e.g. supplier-buyer relationships) by its edges. In this specific field, directed graphs are preferred to undirected ones.

\section{Measures for describing graphs' complexity}

The order of a graph, $n$ and the size of a graph, $m$, i.e. the number of the vertices and the number of the edges, respectively, are natural measures of its complexity. The degree of vertex $v, \operatorname{deg}(v)$ equals the number of edges incident to it.

The entropy of a graph is a more sophisticated measure for graphs' complexity [56,39]. Relying on Shannon's information theory [63], it characterizes the similarity between the vertex degrees in a graph, and is to be derived as follows:

$H_{\text {graph }}=-\sum_{i=1}^{n} \frac{\operatorname{deg}\left(v_{i}\right)}{m} * \log _{2} \frac{\operatorname{deg}\left(v_{i}\right)}{m}$.
The entropy value lies in the interval $\left[0, \log _{2} n\right]$, it is 0 in edgeless graphs and $\log _{2} n$ in fully connected ones.

Measures for describing graphs' robustness

The betweenness centrality of vertex $v$ (also known as vertex betweenness centrality) is the ratio of the number of the shortest paths between vertices that pass $v$ to the total number of the shortest paths in the graph $[64,65]$ :

$B C(v)=\sum_{u \neq w \in V} \frac{\sigma_{u w}(v)}{\sigma_{u w}}$

Here $\sigma_{u w}$ denotes the number of the shortest paths between any vertices $u$ and $w$ while $\sigma_{u w}(v)$ is the number of the shortest paths within this set, which incorporate vertex $v . V$ is the set of all vertices in the graph.

In the interest of making the betweenness centrality of vertices which pertain to graphs of different size comparable, its values are usually divided by factors related to the size of the given graph. In directed graphs, a suitable normalization factor is $(n-1)^{*}(n-2)$, while in undirected ones $(n-1)^{*}(n-2) / 2$. Being directed graphs more adequate to describe supply chains and networks, in this field the former factor is to be used for the determination of the normalized betweenness centrality:

$B C^{\prime}(v)=\frac{B C(v)}{(n-1) *(n-2)}$.

The normalized betweenness centrality of a vertex can take a value in the interval $[0,1]$. The higher this value, the more important the given vertex, which means the graph is less robust here.

The edge betweenness centrality of edge $e$ is also applicable to the analysis of supply chains and networks. It is the ratio of the number of the shortest paths that incorporate the given edge, $\sigma_{u w}(e)$ to the total number of the shortest paths in the graph [64]:

$B C E(e)=\sum_{u \neq w \in V} \frac{\sigma_{u w}(e)}{\sigma_{u w}}$.

A relatively new measure of graphs' robustness is factor $R$ [66,67]:

$R=\frac{1}{n} \sum_{Q=1}^{n} s(Q)$

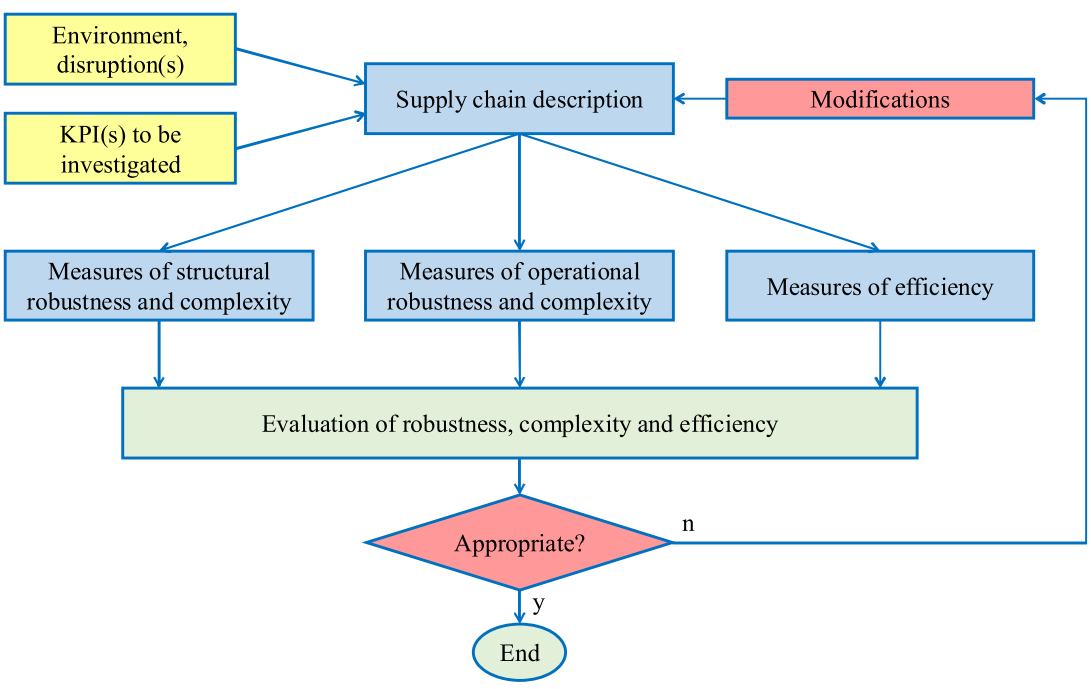

Fig. 2. The framework for evaluating supply chains' robustness, complexity and efficiency [6]. 
where $s(Q)$ is the proportion of the order of the largest connected subgraph remained after removing $Q$ vertices to the order of the original graph. The range of possible $\mathrm{R}$ values lies in the interval $[1 / \mathrm{n}, 1 / 2]$. The endpoints are taken in case of star graphs and fully connected graphs, respectively.

Some other graph measures can also be considered for characterizing the robustness of supply chains and networks, e.g. average shortest distance, average path length and average clustering coefficient [54].

\section{Operational measures of supply chains}

\section{Measures for supply chains' operational complexity}

In addition to the structural complexity, there are very important factors which contribute to the complexity of supply chains:

- Demands: amounts, volatility, seasonality of the demands for different products in different regions.

- Products: number and diversity of products, material types, parts and subassemblies within the supply chain.

- Factories: capacities, the applied production planning and scheduling methods and their parameters, types and parameters of inventory policies, sourcing policies and their parameters.

- Warehouses, distribution centers: capacities, types and parameters of inventory policies, sourcing policies and their parameters.

- Transportation: number and types of vehicles, types of transportation policies.

The above, non-exhaustive list is in harmony with the related literature, see e.g. [68].

\section{Measures for supply chains' operational robustness}

In order to quantify the operational robustness of supply chains, some KPIs have to be defined (Fig. 1).

Those KPIs of supply chains that characterize either the delivery speed or the delivery reliability of orders are of high importance [69]. As examples of the former, the throughput time and the delivery lead time are to be outlined, while as representatives of the latter, the delivery tardiness, the percentage of late deliveries and the service level by orders or by products can be mentioned:

- Throughput time: the average time between the start of an order's production and its completion.

- Delivery lead time: the average time between the placement of an order and its shipment to the customer.
- Delivery tardiness: the average time between the actual and the contractual delivery times, in case of late deliveries.

- Percentage of late deliveries: the proportion of the number of the late deliveries to the number of all deliveries.

- Service level by orders or by products: the proportion of the number of the successful orders to the number of all orders placed, or the proportion of the number of the products in the successful orders to the number of products in all orders placed, respectively.

All these KPIs can be used for characterizing entire supply chains, but in case of a more detailed analysis, they are also appropriate for investigating every single supplier-buyer relationship within them.

The measures of operational robustness are the actual values of the KPIs in focus, or rather their closeness to their anticipated / planned values.

Whereas for the description of the structural properties the use of the graph theory proves to be the most adequate modeling approach, here the statistical methods and simulations can be advantageously applied.

\section{Methodology and framework for the evaluation of supply chains' robustness, complexity and efficiency}

There is a pressing need to investigate the interrelationships of robustness, complexity and efficiency of supply chains in order to support decisions related to their design and management.

On the basis of the considerations and challenges highlighted in Section "Key concepts and challenges", and of the quantitative measures described in Section "Quantitative measures of supply chains' robustness and complexity" for characterizing supply chains' robustness and complexity from both structural and operational points of view, the following methodology for the holistic evaluation of supply chains (including the aspects of robustness, complexity and efficiency) can be proposed:

(1) Definition of the supply chain's environment, together with the disruption(s) and $\operatorname{KPI}(s)$ to be considered.

(2) Description of the supply chain to be analyzed (e.g. product(s) to be produced / delivered, supply chain's structure, capacities of its elements, inventory management policies, production planning and scheduling methods, means of transportation, etc.).

(3) Quantitative characterization of structural properties of the supply chain in respect of both robustness and complexity, based on graph theoretical analysis.
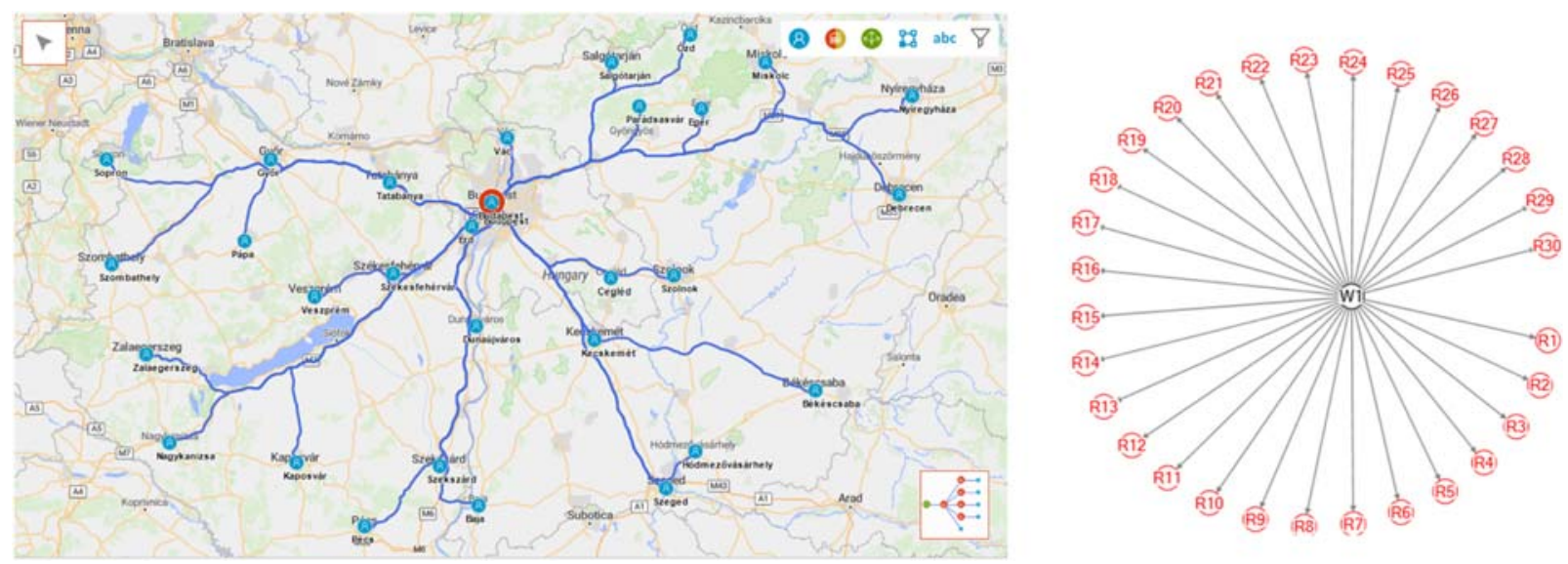

Fig. 3. The starting distribution network and its structure in graph representation. 

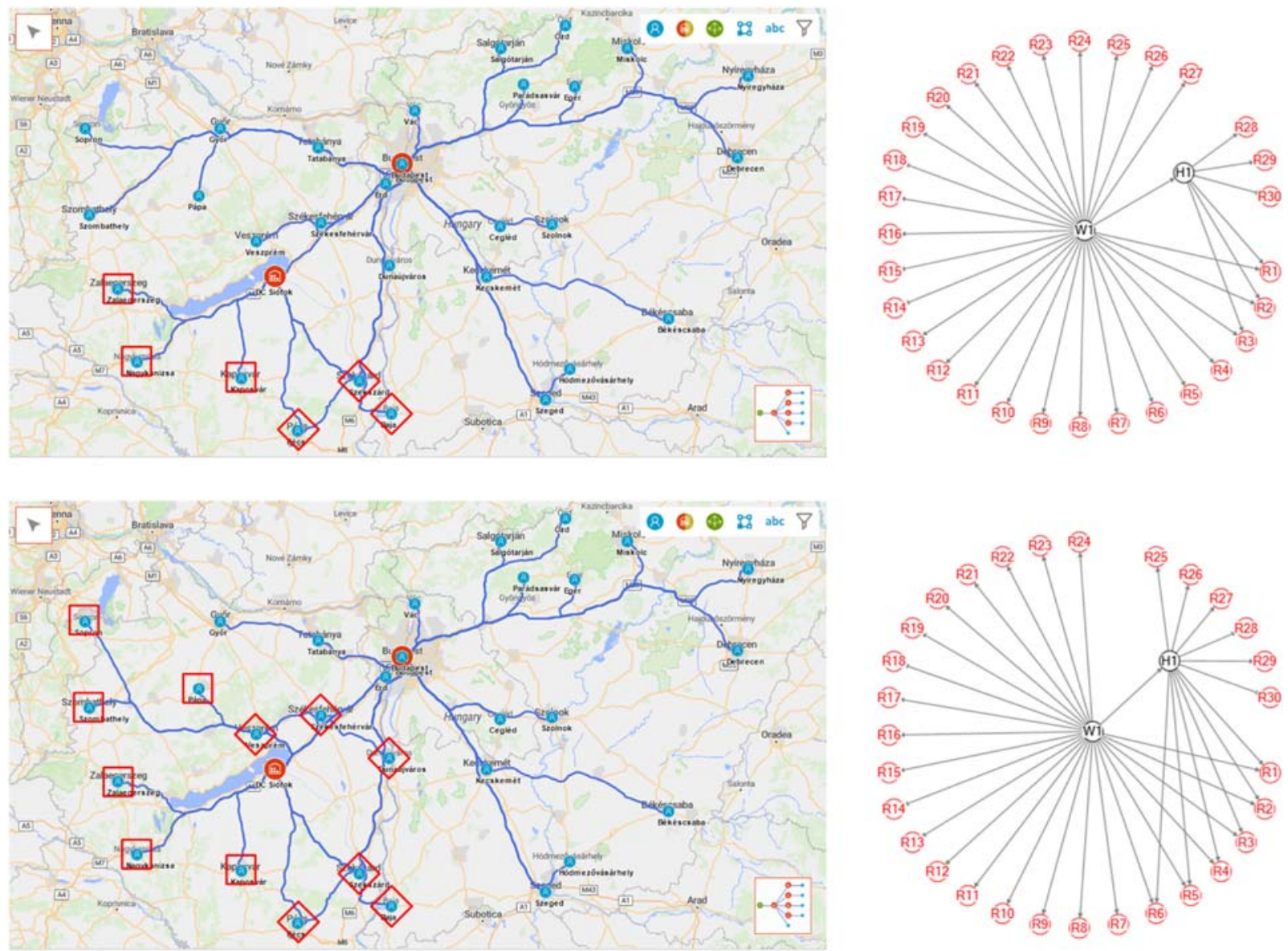

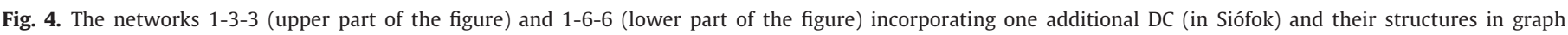
representation.

(4) Quantitative characterization of operational properties of the supply chain also from robustness and complexity points of view, either by analyzing parameters collected from the real system or by supply chain simulation.

(5) Determination of efficiency measures relying on analytical computations or simulation.

(6) Investigation of the appropriateness of the achieved performance. In the negative case go back to Step 2. In order to drive the whole process, searching and optimization techniques can be used.

The framework developed for evaluating robustness, complexity and efficiency of supply chains is illustrated in Fig. 2.

The structural measures are computed partly by the NodeXL network analysis tool (https://www.smrfoundation.org/nodexl/), partly by own algorithms. The operational and the efficiency measures are determined by using the AnyLogistix supply chain software (https://www.anylogistix.com/) offering versatile opportunities for the simulation of supply chains. Simulation is a fundamental approach to the evaluation of supply chain settings. On the one hand, it is not feasible to perform experiments in running supply chains, and on the other, it is nearly impossible to include all the small but many times important details of a supply chain in an analytical model.

By applying the methodology and the framework, different supply chain alternatives can be generated, compared from robustness, complexity and efficiency points of view, and offered for the management. This way, more well-founded decisions can be made, taking all the three aspects and the company's priorities into account.

Here is to be mentioned that the methodology and the framework can be adequately used also for investigating and mitigating the ripple effects in supply chains, as it is illustrated in the next section.

\section{A case study on distribution networks}

In this section, the applicability of the proposed methodology and the developed framework is demonstrated by mitigating the ripple effect in distribution networks through balancing the aspects of robustness, complexity and efficiency.

\section{General description of the distribution networks}

The case study to be reported on here refers to conceived distribution networks situated in Hungary. They comprise 30 regions with demands to be satisfied. In the fundamental setting, the regions are served from a central distribution center (DC) located in Budapest (Fig. 3). (In the graph representations in this section, the regions are denoted by R1-R30, the central DC by W1, and the additional DCs by $\mathrm{H} 1$ and $\mathrm{H} 2$.)

The main operational parameters of the distribution networks are as follows:

- Product: mineral water in 2-liter bottle, its cost and selling price are 0.2 and $0.5 \$ /$ bottle, respectively. 

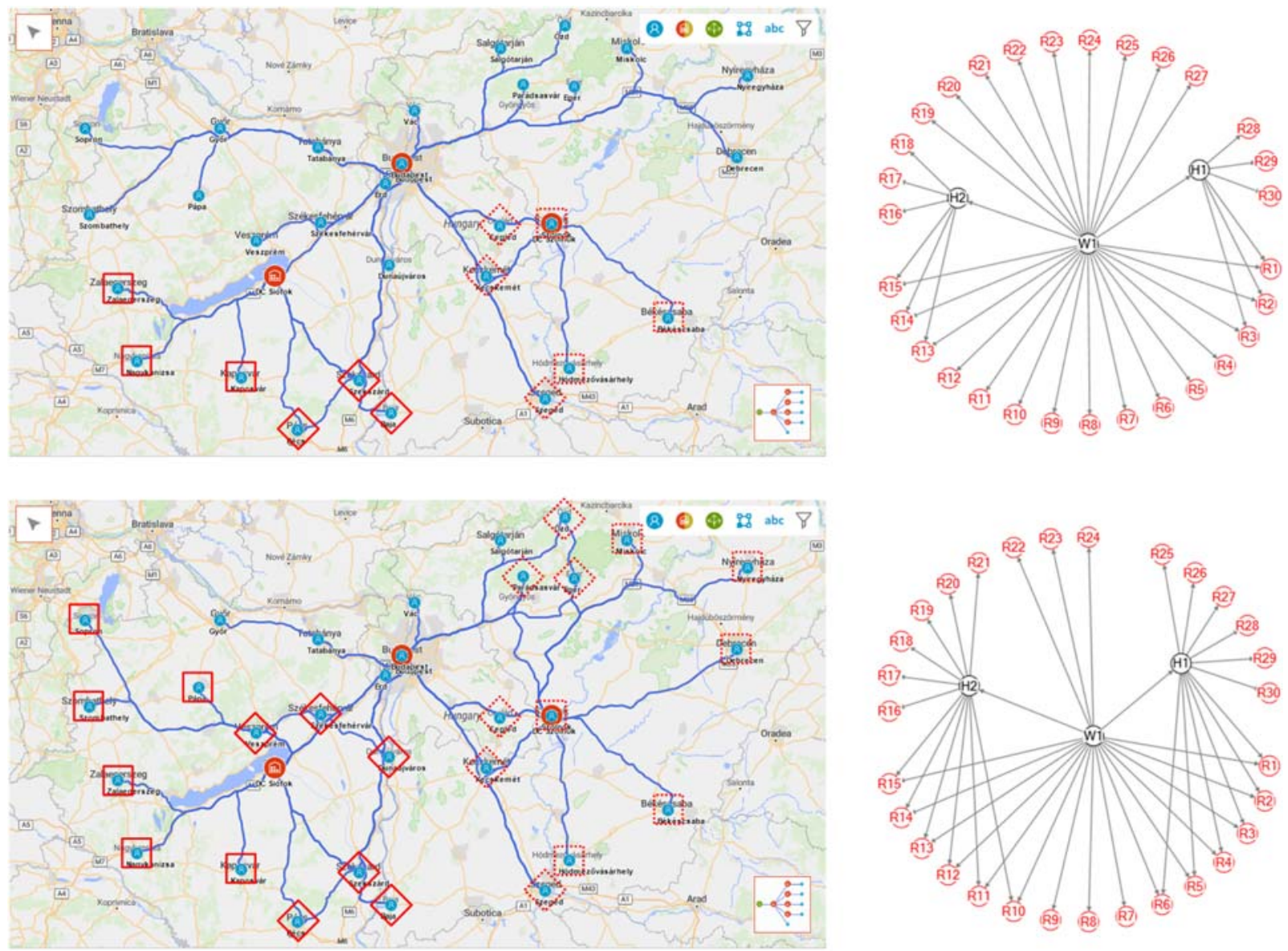

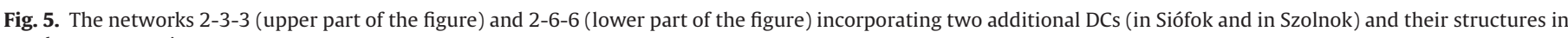
graph representation.

Table 2

Structural complexity and robustness measures.

\begin{tabular}{|c|c|c|c|c|c|c|}
\hline Network structure & No. of nodes & No. of edges & Average degree & Entropy & $\begin{array}{l}\text { Max. of the normalized } \\
\text { betweenness centrality }\end{array}$ & Factor $\mathrm{R}$ \\
\hline $0-0-0$ & 31 & 30 & 0.968 & 3.453 & 1 & 0.032 \\
\hline $1-3-3$ & 32 & 34 & 1.063 & 3.731 & 0.958 & 0.036 \\
\hline $1-6-6$ & 32 & 37 & 1.156 & 3.828 & 0.848 & 0.043 \\
\hline $2-3-3$ & 33 & 38 & 1.152 & 3.965 & 0.921 & 0.047 \\
\hline $2-6-6$ & 33 & 44 & 1.333 & 4.111 & 0.716 & 0.063 \\
\hline
\end{tabular}

- Demands: deterministic, constant over time, proportional to the number of inhabitants in the given regions, 0.4 liter/day/ inhabitant.

- Order parameters of the regions: order interval: 5 days, expected lead time (ELT): 7 days, backorder is not allowed.

- Parameters of the DCs: carrying cost: $0.001 \$ / \mathrm{m}^{3} /$ day, initial cost of an additional DC: 1 million \$, the additional DCs use min-max inventory policy $(\mathrm{s}, \mathrm{S})$ with a periodic check of 1 day.

- Transportation: less than truckload (LTL) policy, trucks with capacity $50 \mathrm{~m}^{3}$ and speed $60 \mathrm{~km} / \mathrm{h}$, with cost $0.05 \$ / \mathrm{m}^{3} / \mathrm{km}$ calculated with actual routes (not with straight lines).

The investigated time period is 1 calendar year, with a 1 -month disruption at the central DC in Budapest (the DC is temporarily closed).

The next two subsections illustrate how structural and operational modifications can result in distribution network alternatives with improved ripple effect mitigation abilities.

\section{Structural modifications}

Obviously, the star structure in Fig. 3 is extremely vulnerable. The structure was augmented step by step: first, one additional DC (in Siófok) was added to the network, which was supplied from the DC in Budapest. Three of the regions (Zalaegerszeg, Nagykanizsa and Kaposvár, indicated by squares with horizontal and vertical edges) were served exclusively from Siófok, three other regions (Pécs, Szekszárd and Baja, marked by squares standing on their vertices) from both Budapest and Siófok (in equal ratio, in cases when both of them were functioning), and the remaining 24 regions solely from Budapest (Fig. 4, upper part). (Generally, the network structures analyzed here can be described with the triple $a-b-c$ where $a$ is the number of additional DC(s), each of which supplies $b$ regions jointly with the central DC, and $c$ regions exclusively.)

The next network was differentiated from the preceding one only in the numbers of how many regions were served solely by 

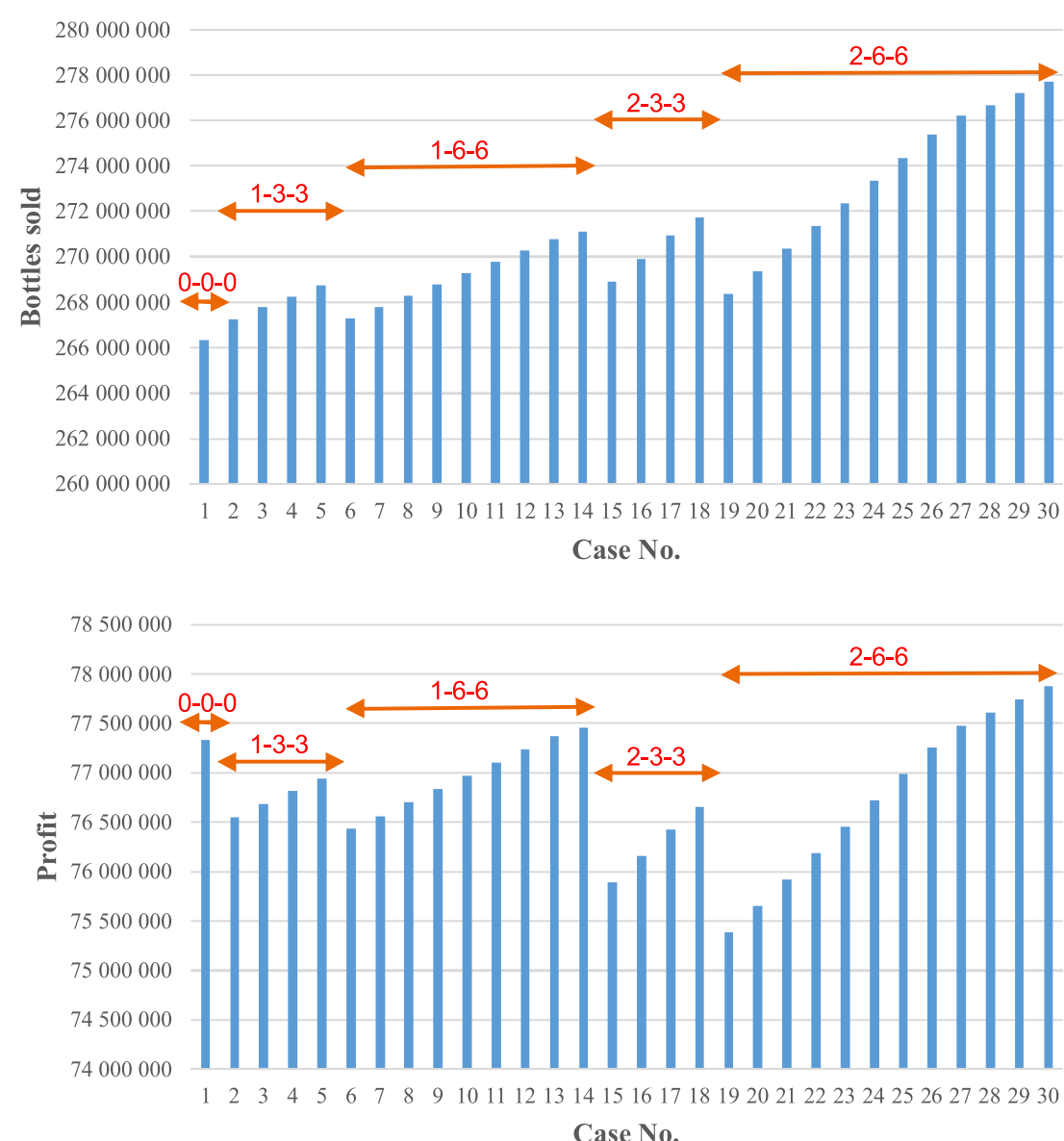

Fig. 6. Number of bottles sold and profit (in \$).

Siófok, and how many by Budapest and Siófok together (similar graphical indications are used as before). These numbers were 6-6 (Fig. 4, lower part).

Finally, two other networks were generated both having two additional DCs (in Siófok and in Szolnok). Similarly to the cases with one additional DC, these DCs served some (3 and 6) regions exclusively, and the same numbers jointly with Budapest (Fig. 5). (The newly involved regions are marked by squares with dotted edges.)

Some structural complexity and robustness measures defined in Subsection "Structural measures of supply chains" were determined for the five networks (Table 2) by using the framework introduced in Section "Methodology and framework for the evaluation of supply chains' robustness, complexity and efficiency". As described there, the structural measures were computed partly by the NodeXL network analysis tool, partly by own algorithms.

Comparing the values of the complexity measures (number of the nodes, number of the edges, average degree of the nodes, entropy of the graph (see Subsection "Measures for describing graphs' complexity")) in rows 2-5 (networks 1-3-3, 1-6-6, 2-3-3 and 2-6-6), with the values of the basic distribution network owning only one, central DC (0-0-0, first row), it can be seen that all measures exceed their initial values. Within the blocks of networks with the same number of additional DCs (1-3-3, 1-6-6 and 2-3-3, 2$6-6$, respectively), the increase is monotonous. It is also worthy of note that - as a result of the more and more evenly distributed node degrees in the structures - the monotonous increase of the entropy values is experienced for all the consecutive networks.
Looking at the robustness-related measures (maximum of the normalized betweenness centrality, factor $\mathrm{R}$ (see Subsection "Measures for describing graphs' robustness")) in the two righthand side columns, it can be observed that in case of the networks belonging to the same block, their augmentation with further edges led to growing robustness measures. This outcome is in harmony with the general perception that if a higher proportion of the regions applies multiple sourcing, the networks' robustness increases.

\section{Operational modifications}

The coming part shows how the different distribution networks behave in case of the disruption, taking not only structural but also operational parameters into account.

In the example under discussion, the disruption occurs at the central DC. It causes ripples, its negative effects gradually spread across the whole distribution network, from the central DC, through the additional DCs, and finally to the regions. Obviously, the additional DCs play a crucial role in mitigating the ripple effect, and consequently, a logical way was to concentrate on their inventory levels.

Numerous possible KPIs, e.g. number of bottles sold, costs related to inventory and transportation, revenue, profit, and service levels, were determined through simulation by applying the framework highlighted in Section "Methodology and framework for the evaluation of supply chains' robustness, complexity and efficiency". As outlined there, for this purpose, the AnyLogistix supply chain software was used. Here, three KPIs are analyzed and 

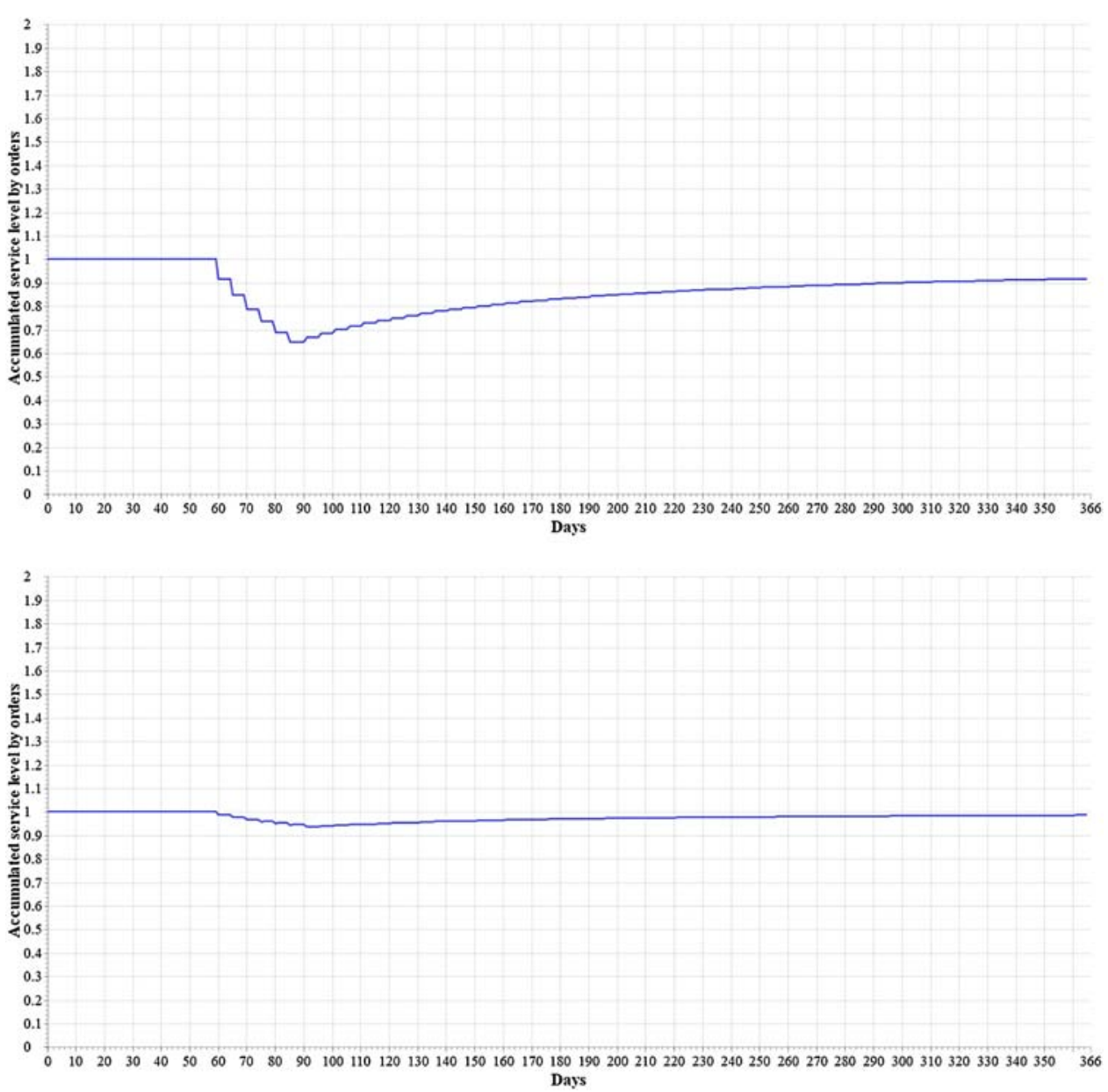

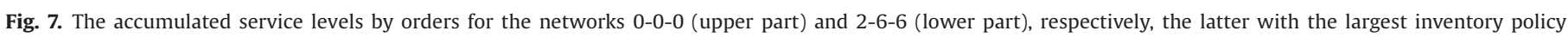
parameters considered.

illustrated, namely the number of bottles sold and the profit (Fig. 6), and the accumulated service level by orders (Fig. 7).

The triples in Fig. 6 indicate the network structures, as in Subsection "Structural modifications". Altogether 30 cases were considered, 1 in the basic structure, 4, 9, 4 and 12 in structures 1-3$3,1-6-6,2-3-3$ and 2-6-6, respectively. Within the sets with the same structural complexity, the consecutive cases incorporated enhanced operational complexity, by increasing the parameters of the min-max inventory policy of the additional DCs step by step. In each step, the min (s) and the max (S) parameters were increased by the same amount (500 000 bottles).

As expected, the smallest amount of mineral water was sold in the basic network with no additional DC. The amount monotonically increased within each set, in parallel with the networks' enhanced operational complexity.

Looking at the profit (as efficiency measure), one can see that the inclusion of the additional DCs having initial cost, in most cases resulted in decreased profitability. Generally, this phenomenon is called "the cost of robustness" [14]. However, in the networks with 1-6-6 and 2-6-6 structures, with relatively large inventory policy parameters (cases 13 and 14, and cases 27-30, respectively), the profit could even surpass the value yielded by the reference network.

To the service level of a network similar importance can be attached as to its profitability. Considering the 1-year period, the accumulated service level by orders (as robustness measure) increased monotonically set by set, starting with 0.92 (0-0-0, case 1) and ending with 0.98 (2-6-6, case 30$)$. As to these two extreme cases, Fig. 7 shows the accumulated service levels by orders for the period investigated, assuming the disruption being in March.

\section{Interpretation of the results}

The disruption considered in the case study lasted for one month at the central DC in Budapest. Throughout that period, no region could be served directly from this DC. Obviously, modifications had to be initiated in order to alleviate the consequences of the temporary shutdown.

The question was how to balance the aspects of robustness, complexity and efficiency while mitigating the ripple effect of this disruption on the other parts of the investigated distribution networks. For this purpose, several strategies were implemented, and their impacts were analyzed. The strategies were as follows: (1) the augmentation of the starting distribution network with additional DC(s), (2) the use of multiple sourcing in different extents, both as structural modifications; (3) the step-by-step increase of the min-max inventory policy parameters of the additional DC(s), as operational modifications.

The structural robustness measures of the starting distribution network pointed out that the network structure was extremely vulnerable to potential disruptions at the central DC. This situation could be significantly improved by the structural modifications, which went hand in hand with the increase of the structural complexity measures (Table 2). Appropriate combinations of the structural and the operational modifications led to distribution network alternatives that represented balanced solutions between 
the aspects of robustness, complexity and efficiency, and, on the basis of the considered KPIs (Figs. 6 and 7), could count on the management's satisfaction.

\section{Conclusions}

In the paper, robustness and complexity of supply chains were treated jointly, not restricted exclusively to their structural or operational aspects, but considering both. Moreover, the complicated interrelationships of robustness, complexity and efficiency were put into the focus.

For the sake of unambiguity and understandability, relying on a comprehensive literature analysis of previous works, key concepts and challenges related to the content of the paper were defined and commented on:

- On the basis of their occurrence probabilities and potential impacts, two risk categories were identified as highly challenging for the researchers: the recurrent (sometimes called operational) risks and the disruptive ones, i.e. frequent events with low impact and rare events with high impact, respectively.

- It was outlined that comparing with the relatively well-known bullwhip effect, which belongs to the recurrent risk category, in the literature generally less attention was given to the ripple effect, a representative of the disruptive risk category.

- A number of terms connected to robustness were listed, e.g. resilience, agility, responsiveness, flexibility, changeability and vulnerability, and furthermore, a comprehensive robustness definition was formulated and used in the paper.

- Dealing with the seemingly irreconcilable conflicts between efficiency and robustness, the target to balance their opposing characteristics was set.

- As to the relation of supply chains' complexity and robustness, the challenge to achieve the required level of robustness with the lowest possible level of complexity was formulated.

- Finally, research gaps were identified, and this way the timeliness of the research was justified.

Aiming to balance the aspects of robustness, complexity and efficiency in supply chains, and, necessarily, to compare different supply chain settings in a reliable way, measures - primarily quantitative ones - have to be defined. For efficiency there are a number of well-known measures available, however this is not the case for robustness and complexity. In the paper, graph theoretical measures were given for characterizing the structural robustness and complexity of supply chains and networks. Measures for assessing their operational robustness and complexity were also highlighted, outlining that in this field statistical methods and simulation techniques are more adequate.

A methodology and a framework for the holistic evaluation of supply chains' robustness, complexity and efficiency were depicted. Their applicability was illustrated by the results of the case study described in the paper, demonstrating how the envisioned balance between the three aspects can be achieved while mitigating the ripple effect in distribution networks.

It was shown that with appropriate changes in both the structural and the operational complexity, the robustness can be significantly enhanced, while sometimes maintaining - rarely even increasing the level of profitability.

In addition to the scientific novelty of the proposed approach which is in line with the main tendencies of supply chain and production network research [59,70], it has clear practical relevance too. The latter may even further increase in the era of natural disasters and pandemics [62]. The methodology and the framework can be advantageously used in the (re)design, analysis and management of supply chains, and can be made capable of acting as a digital twin of them.

Further work has been initiated for extending the investigations to multitier supply chains and networks.

\section{Declaration of interests}

The author declares that she has no known competing financial interests or personal relationships that could have appeared to influence the work reported in this paper.

\section{Acknowledgements}

The research has been supported by the GINOP-2.3.2-15-201600002 and the NKFIA ED_18-22018-0006 grants.

\section{References}

[1] Ivanov, D., Dolgui, A., 2020, Viability of intertwined supply networks: Extending the supply chain resilience angles towards survivability. A position paper motivated by COVID-19 outbreak. International Journal of Production Research, 58/10: 2904-2915.

[2] Monostori, L., Váncza, J., 2020, Lessons learned from the COVID-19 pandemic and their possible consequences on manufacturing. Smart and Sustainable Manufacturing Systems, 4/3: 333-337.

3] Belhadi, A., Kamble, S., Chiappetta Jabbour, C.J., Gunasekaran, A., Ndubisi, N.O. Venkatesh, M., 2021, Manufacturing and service supply chain resilience to the COVID-19 outbreak: Lessons learned from the automobile and airline industries. Technological Forecasting \& Social Change, 163:1-19. Paper No. 120447

[4] El Baz, J., Ruel, S., 2021, Can supply chain risk management practices mitigate the disruption impacts on supply chains' resilience and robustness? Evidence from an empirical survey in a COVID-19 outbreak era. International Journal of Production Economics, 233:1-12. Paper No. 107972.

[5] Monostori, L., Kádár, B., Bauernhansl, T., Kondoh, S., Kumara, S.R.T., Reinhart, G., Sauer, O., Schuh, G., Sihn, W., Ueda, K., 2016, Cyber-physical systems in manufacturing. CIRP Annals - Manufacturing Technology, 65/2: 621-641.

[6] Monostori, J., 2018, Supply chains' robustness: Challenges and opportunities. Procedia CIRP, 67:110-115.

[7] Monostori, J., 2016, Robustness- and complexity-oriented characterization of supply networks' structures. Procedia CIRP, 57:67-72.

[8] Wagner, S.M., Bode, C., 2006, An empirical investigation into supply chain vulnerability. Journal of Purchasing and Supply Management, 12/6: 301-312.

[9] Vilko, J.P.P., Hallikas, J.M., 2012, Risk assessment in multimodal supply chains. International Journal of Production Economics, 140/2: 586-595.

[10] Tomlin, B., 2006, On the value of mitigation and contingency strategies for managing supply chain disruption risks. Management Science, 52/5: 639-657.

[11] Dolgui, A., Ivanov, D., Sokolov, B., 2018, Ripple effect in the supply chain: An analysis and recent literature. International Journal of Production Research, 56/ 1-2: 414-430

[12] Forrester, J.W., 1961, Industrial Dynamics. MIT Press, Cambridge, Massachusetts, USA.

[13] Ivanov, D., Dolgui, A., Sokolov, B., 2015, Supply chain design with disruption considerations: Review of research streams on the ripple effect in the supply chain. IFAC-PapersOnLine, 48/3: 1700-1707.

[14] Ivanov, D., 2018, Structural Dynamics and Resilience in Supply Chain Risk Management. Springer, Cham, Switzerland.

[15] Ferdows, K., 1997, Making the most of foreign factories. Harvard Business Review, March-April: 73-88.

[16] Asbjornslett, B.E., Rausand, M., 1999, Assess the vulnerability of your production system. Production Planning \& Control, 10/3: 219-229.

[17] Bundschuh, M., Klabjan, D., Thurston, D.L., 2006, Modeling Robust and Reliable Supply Chains, Working Paper. University of Illinois at Urbana-Champaign, Illinois, USA

[18] Dong, M., 2006, Development of supply chain network robustness index. International Journal of Services Operations and Informatics, 1/1/2: 54-66.

[19] Dong, M., Chen, F.F., 2007, Quantitative robustness index design for supply chain networks. in Jung H. , Jeong B. , Chen F.F , (Eds.) Trends in Supply Chain Design and Management - Technologies and Methodologies. Springer-Verlag London, UK369-391.

[20] Vlajic, J.V., van der Vorst, J.G.A.J., Haijema, R., 2012, A framework for designing robust food supply chains. International Journal of Production Economics, 137 1: $176-189$.

[21] Sheffi, Y., 2007, The Resilient Enterprise: Overcoming Vulnerability for Competitive Advantage. MIT Press, Cambridge, Massachusetts, USA

[22] Christopher, M., Peck, H., 2004, Building the resilient supply chain. The International Journal of Logistics Management, 15/2: 1-14.

[23] Ponomarov, S.Y., Holcomb, M.C., 2009, Understanding the concept of supply chain resilience. The International Journal of Logistics Management, 20/1 124-143. 
[24] Barroso, A.P., Machado, V.H., Cruz-Machado, V., 2011, Supply chain resilience using the mapping approach. in Li P., (Ed.) Supply Chain Management. IntechOpen, London, UK. pp. 161-184.

[25] Bhamra, R., Dani, S., Burnard, K., 2011, Resilience: The concept, a literature review and future directions. International Journal of Production Research, 49/ 18: 5375-5393.

[25] Bhamra, R., Dani, S., Burnard, K., 2011, Resilience: The concept, a literature review and future directions. International Journal of Production Research, 49/ 18: 5375-5393.

[26] Kamalahmadi, M., Mellat Parast, M., 2016, A review of the literature on the principles of enterprise and supply chain resilience: Major findings and directions for future research. International Journal of Production Economics, 171:116-133.

[27] Váncza, J., Monostori, L., Lutters, D., Kumara, S.R.T., Tseng, M., Valckenaers, P., Van Brussel, H., 2011, Cooperative and responsive manufacturing enterprises. CIRP Annals - Manufacturing Technology, 60/2: 797-820.

[28] Carvalho, H., Azevedo, S.G., Cruz-Machado, V., 2012, Agile and resilient approaches to supply chain management: Influence on performance and competitiveness. Logistics Research, 4:49-62.

[29] Wieland, A., Wallenburg, C.M., 2013, The influence of relational competencies on supply chain resilience: A relational view. International Journal of Physical Distribution \& Logistics Management, 43/4: 300-320.

[30] Stricker, N., Lanza, G., 2014, The concept of robustness in production systems and its correlation to disturbances. Procedia CIRP, 19:87-92.

[31] Asbjornslett, B.E., 2009, Assessing the vulnerability of supply chains. in Zsidisin G.A, Ritchie B, (Eds.) Supply Chain Risk - A Handbook of Assessment, Management, and Performance. Springer, USA . pp. 15-33.

[32] Ivanov, D., Dolgui, A., 2019, Low-certainty-need (LCN) supply chains: A new perspective in managing disruption risks and resilience. International Journal of Production Research, 57/15-16: 5119-5136.

[33] Fiksel, J., Polyviou, M., Croxton, K.L., Pettit, T.J., 2015, From risk to resilience: Learning to deal with disruption. MIT Sloan Management Review, 56/2: 79-86.

[34] Wiendahl, H.-P., Scholtissek, P., 1994, Management and control of complexity in manufacturing. CIRP Annals - Manufacturing Technology, 43/2: 533-540.

[35] ElMaraghy, W., ElMaraghy, H., Tomiyama, T., Monostori, L., 2012, Complexity in engineering design and manufacturing. CIRP Annals - Manufacturing Technology, 61/2: 793-814.

[36] Choi, T.Y., Krause, D.R., 2006, The supply base and its complexity: Implications for transaction costs, risks, responsiveness, and innovation. Journal of Operations Management, 24:637-652.

[37] Serdarasan, S., 2013, A review of supply chain complexity drivers. Computers \& Industrial Engineering, 66/3: 533-540.

[38] Tolio, T., Urgo, M., Váncza, J., 2011, Robust production control against propagation of disruptions. CIRP Annals - Manufacturing Technology, 60/1: 489-492.

[39] Cheng, C.-Y., Chen, T.-L., Chen, Y.-Y., 2014, An analysis of the structural complexity of supply chain networks. Applied Mathematical Modelling, 38/9-10: 2328-2344.

[40] Stricker, N., Pfeiffer, A., Moser, E., Kádár, B., Lanza, G., Monostori, L., 2015, Supporting multi-level and robust production planning and execution. CIRP Annals - Manufacturing Technology, 64/1: 415-418.

[41] Wagner, S.M., Neshat, N., 2010, Assessing the vulnerability of supply chains using graph theory. International Journal of Production Economics, 126/1: 121-129.

[42] Zhao, K., Kumar, A., Yen, J., 2011, Achieving high robustness in supply distribution networks by rewiring. IEEE Transactions on Engineering Management, 58/2: 347-362.

[43] Gutiérrez-Pérez, J.A., Herrera, M., Pérez-García, R., Ramos-Martínez, E., 2013. Application of graph-spectral methods in the vulnerability assessment of water supply networks. Mathematical and Computer Modelling, 57/7-8: 1853-1859.

[44] Bates, S., Angeon, V., Ainouche, A., 2014, The pentagon of vulnerability and resilience: A methodological proposal in development economics by using graph theory. Economic Modelling, 42:445-453.

[45] Nakatani, J., Tahara, K., Nakajima, K., Daigo, I., Kurishima, H., Kudoh, Y., Matsubae, K., Fukushima, Y., Ihara, T., Kikuchi, Y., Nishijima, A., Moriguchi, Y., 2018, A graph theory-based methodology for vulnerability assessment of supply chains using the life cycle inventory database. Omega, 75:165181.
[46] Tan, W.J., Zhang, A.N., Cai, W., 2019, A graph-based model to measure structural redundancy for supply chain resilience. International Journal of Production Research, 57/20: 6385-6404.

[47] Sivadasan, S., Efstathiou, J., Calinescu, A., Huaccho Huatuco, L., 2006, Advances on measuring the operational complexity of supplier-customer systems. European Journal of Operational Research, 171/1: 208-226.

[48] Huaccho Huatuco, L., Efstathiou, J., Calinescu, A., Sivadasan, S., Kariuki, S., 2009, Comparing the impact of different rescheduling strategies on the entropicrelated complexity of manufacturing systems. International Journal of Production Research, 47/15: 4305-4325.

[49] Isik, F., 2010, An entropy-based approach for measuring complexity in supply chains. International Journal of Production Research, 48/12: 3681-3696.

[50] Holland, J.H., 1995, Hidden Order: How Adaptation Builds Complexity. Helix Books, Addison-Wesley, Reading, Massachusetts, USA.

[51] Choi, T.Y., Dooley, K.J., Rungtusanatham, M.J., 2001, Supply networks and complex adaptive systems: Control versus emergence. Journal of Operations Management, 19:351-366.

[52] Surana, A., Kumara, S.R.T., Greaves, M., Raghavan, U.N., 2005, Supply-chain networks: A complex adaptive systems perspective. International Journal of Production Research, 43/20: 4235-4265.

[53] Pathak, S.D., Day, J.M., Nair, A., Sawaya, W.J., Kristal, M.M., 2007, Complexity and adaptivity in supply networks: Building supply network theory using a complex adaptive systems perspective. Decision Sciences, 38/4: 547-580.

[54] Cui, L.Y., Kumara, S.R.T., Albert, R., 2010, Complex networks: An engineering view. IEEE Circuits and Systems Magazine, 10/3: 10-25.

[55] Kito, T., Ueda, K., 2014, The implications of automobile parts supply network structures: A complex network approach. CIRP Annals - Manufacturing Technology, 63/1: 393-396.

[56] Modrak, V., Marton, D., 2012, Modelling and complexity assessment of assembly supply chain systems. Procedia Engineering, 48:428-435.

[57] Olivares Aguila, J., ElMaraghy, W., 2018, Structural complexity and robustness of supply chain networks based on product architecture. International Journal of Production Research, 56/20: 6701-6718.

[58] Craighead, C.W., Blackhurst, J., Rungtusanatham, M.J., Handfield, R.B., 2007, The severity of supply chain disruptions: Design characteristics and mitigation capabilities. Decision Sciences, 38/1: 131-156.

[59] Ivanov, D., Sokolov, B., 2013, Control and system-theoretic identification of the supply chain dynamics domain for planning, analysis and adaptation of performance under uncertainty. European Journal of Operational Research, 224/2: 313-323.

[60] Cardoso, S.R., Barbosa-Póvoa, A.P., Relvas, S., Novais, A.Q., 2015, Resilience metrics in the assessment of complex supply-chains performance operating under demand uncertainty. Omega, 56:53-73.

[61] Bode, C., Wagner, S.M., 2015, Structural drivers of upstream supply chain complexity and the frequency of supply chain disruptions. Journal of Operations Management, 36:215-228.

[62] Monostori, J., 2020, Beyond the profit motive: Environmentally conscious (re)design of supply chain structures. Procedia CIRP, 93:808-813.

[63] Shannon, C.E., Weaver, W., 1971, The Mathematical Theory of Communication. University of Illinois Press, Urbana-Champaign, Illinois, USA.

[64] Holme, P., Kim, B.J., Yoon, C.N., Han, S.K., 2002, Attack vulnerability of complex networks. Physical Review E, 65/5: 1-15. Paper No. 056109.

[65] Duan, Y., Lu, F., 2014, Robustness of city road networks at different granularities. Physica A: Statistical Mechanics and its Applications, 411:21-34.

[66] Schneider, C.M., Moreira, A.A., Andrade, J.S., Havlin, S., Herrmann, H.J., 2011, Mitigation of malicious attacks on networks. Proceedings of the National Academy of Sciences of the United States of America PNAS, 108/10: 3838-3841.

[67] Zhou, M., Liu, J., 2014, A memetic algorithm for enhancing the robustness of scale-free networks against malicious attacks. Physica A: Statistical Mechanics and its Applications, 410:131-143.

[68] Bozarth, C.C., Warsing, D.P., Flynn, B.B., Flynn, E.J., 2009, The impact of supply chain complexity on manufacturing plant performance. Journal of Operations Management, 27/1: 78-93.

[69] Vachon, S., Klassen, R.D., 2002, An exploratory investigation of the effects of supply chain complexity on delivery performance. IEEE Transactions on Engineering Management, 49/3: 218-230.

[70] Lanza, G., Ferdows, K., Kara, S., Mourtzis, D., Schuh, G., Váncza, J., Wang, L., Wiendahl, H.-P., 2019, Global production networks: Design and operation. CIRP Annals - Manufacturing Technology, 68/2: 823-841. 